\title{
Acoustic Emission Source Linear Localization Based on an Ultra-Short FBGs Sensing System
}

\author{
Zhongwei JIN, Mingshun JIANG ${ }^{*}$, Qingmei SUI, Faye ZHANG, and Lei JIA \\ School of Control Science and Engineering, Shandong University, Jinan, 250061, China \\ *Corresponding author: Mingshun JIANGＥmail: sdujiangmingshun@163.com
}

\begin{abstract}
An acoustic emission (AE) linear location system was proposed, which employed fiber Bragg gratings (FBGs) as AE sensors. It was demonstrated that the FBG wavelength could be modulated as the static case when the grating length was much shorter than the AE wavelength. In addition, an improved AE location method based on the Gabor wavelet transform (WT) and threshold analysis was represented. The method was testified through AE linear location experiments based on a tunable narrow-band laser interrogation system using ultra-short FBG sensors as AE sensors. Results of the experiments showed that $86 \%$ of the linear location errors were less than $10 \mathrm{~mm}$.
\end{abstract}

Keywords: Acoustic emission (AE) linear location, fiber Bragg gratings (FBGs), Gabor wavelet transform (WT), threshold analysis

Citation: Zhongwei JIN, Mingshun JIANG, Qingmei SUI, Faye ZHANG, and Lei JIA, “Acoustic Emission Source Linear Localization Based on an Ultra-Short FBGs Sensing System," Photonic Sensors, 2014, 4(2): 152-155.

\section{Introduction}

The fiber Bragg grating (FBG) sensor has been expected as an ideal candidate for acoustic emission (AE) detection, because FBGs are not susceptible to the electromagnetic field, have multifunctionality, and enable multiplexing $[1,2]$. In previous study, Aldo Minardo et al. found the actual influence of ultrasonic wave (US) perturbation on the shape and central wavelength of the grating peak [3]. Jung-Ryul Lee et al. designed an FBG AE sensor for mechanical tests and demonstrated that the ultrasonic vibration could propagate along the optical fiber with low attenuation [4-6]. The AE source location is an effective technique in structure health monitoring and has attracted great interest among researchers. Kwon et al. provided a source location method using the inverse wavelet transform
[7]. Jeong et al. reported a location method using the wavelet transform based on the Gabor wavelet to distinguish the different modes of the $\mathrm{AE}$ wave [8]. In this paper, the response of FBGs to $\mathrm{AE}$ waves is theoretically analyzed first, and the FBG based AE sensors and the interrogation system based on a tunable narrow-band laser are designed. An improved $\mathrm{AE}$ location method based on the Gabor wavelet transform (WT) and threshold analysis is deduced. And finally, an AE linear location system and experiments are conducted.

\section{Theory}

\subsection{Principle of FBGs response to $\mathrm{AE}$ wave}

The reflective wavelength of the FBG under matched condition is defined as

$$
\lambda_{B 0}=2 n_{\text {effo }} \Lambda_{0}
$$

Received: 3 November 2013 / Revised version: 8 December 2013

(C) The Author(s) 2014. This article is published with open access at Springerlink.com

DOI: $10.1007 / \mathrm{s} 13320-014-0176-1$

Article type: Regular 
where $\lambda_{B 0}$ is the reflective wavelength of the FBG, $n_{\text {effo } 0}$ is the effective refractive index of the fiber core, and $\Lambda_{0}$ is the period of the gratings. Both $n_{\text {eff } 0}$ and $\Lambda_{0}$ will be changed by environmental conditions, such as temperature, strain, and $\mathrm{AE}$ waves. When the $\mathrm{AE}$ wave travels along the fiber axis, for $\lambda_{s} / L>>1$, the reflective wavelength of the FBG can be expressed as

$$
\Delta \lambda_{0}=\lambda_{B 0} \varepsilon_{m}\left\{1-\left(\frac{n_{\text {effo }}^{2}}{2}\right) \cdot\left[P_{12}-v\left(P_{11}+P_{12}\right)\right]\right\}
$$

where $\varepsilon_{m}$ denotes the displacement amplitude of the $\mathrm{AE}$ wave, and $\Delta \lambda_{0}$ is the amplitude of the FBG wavelength modulation induced by the $\mathrm{AE}$ waves. From (2), we can see that under the condition of $\lambda_{s} / L>>1$, the grating response in terms of a central wavelength shift is the same as that in the static case [9].

\subsection{Frequency analysis of $\mathrm{AE}$ wave using Gabor based WT}

$\mathrm{AE}$ waves propagating in plate-like structures mainly contain two wave modes: the flexural wave $\left(\mathrm{A}_{0}\right)$ with the low frequency and high amplitude and the extensional wave $\left(\mathrm{S}_{0}\right)$ with the high frequency and low amplitude. In order to improve the accuracy of the AE source location, we can extract a single frequency component from the raw $\mathrm{AE}$ signal using the wavelet transform. Supposing an AE wave containing two harmonic unit waves with slightly different frequencies $\omega_{1}$ and $\omega_{2}$ propagates in the $x$-direction in certain plate-like structure, i.e.:

$$
u(x, t)=e^{-i\left(k_{1} x-\omega_{1} t\right)}+e^{-i\left(k_{2} x-\omega_{2} t\right)} .
$$

Introducing

$$
\begin{aligned}
& \left(k_{1}+k_{2}\right) / 2=k_{c} ;\left(\omega_{1}+\omega_{2}\right) / 2=\omega_{c} \\
& \left(k_{1}-k_{2}\right) / 2=\Delta k ;\left(\omega_{1}-\omega_{2}\right) / 2=\Delta \omega
\end{aligned},
$$

then, (4) can be rewritten as

$$
u(x, t)=2 \cos (\Delta k x-\Delta \omega t) e^{-i\left(k_{c} x-\omega_{c} t\right)} .
$$

The Gabor wavelet has been proved by researchers to be the most suitable wavelet for discrimination of AE modes [8]. Hence, we use the Gabor wavelet as the analyzing wavelet, and the WT of $u(x, t)$ can be obtained as

$$
\begin{aligned}
& (W u)(x, a, b)=\sqrt{a}\left[e^{-i\left(k_{1} x-\omega_{1} b\right)} \overline{\hat{\psi}_{g}\left(a \omega_{1}\right)}\right. \\
& \left.+e^{-i\left(k_{2} x-\omega_{2} b\right)} \overline{\hat{\psi}_{g}\left(a \omega_{2}\right)}\right] .
\end{aligned}
$$

And the magnitude of the WT is

$$
\begin{aligned}
& |(W u)(x, a, b)|=\sqrt{a}\left\{\left[\hat{\psi}_{g}\left(a \omega_{1}\right)\right]^{2}+\left[\psi_{g}\left(a \omega_{2}\right)\right]^{2}\right. \\
& \left.+2 \hat{\psi}_{g}\left(a \omega_{1}\right) \psi_{g}\left(a \omega_{2}\right) \cos (2 \Delta k x-2 \Delta \omega b)\right\}^{1 / 2} .
\end{aligned}
$$

If $\Delta \omega$ is small enough such that $\hat{\psi}_{g}^{\wedge}\left(a \omega_{1}\right) \approx \psi_{g}\left(a \omega_{2}\right) \approx \psi_{g}\left(a \omega_{c}\right)$, we can obtain $|(W u)(x, a, b)| \approx \sqrt{2 a}\left|\hat{\psi}_{g}\left(a \omega_{c}\right)\right|[1+\cos (2 \Delta k x-2 \Delta \omega b)]^{1 / 2}$.

The result shows that $|(W u)(x, a, b)|$ gets its maximum value when $a=\omega_{0} / \omega_{c}$ and $b=(\Delta k / \Delta \omega) x=x / c_{g}$. That is, for the fixed $\boldsymbol{x}$, the magnitude of the WT reveals a peak at $a=\omega_{0} / \omega_{c}$ and $b=(\Delta k / \Delta \omega) x=x / c_{g}$ on the time-frequency plane. Therefore, the peak of the magnitude of the WT corresponds to the propagation of a wave with the group velocity at the frequency $\omega_{c}[8]$.

\section{Experiments and results}

A schematic of the experimental setup is shown in Fig. 1, which is a narrow-band tunable laser interrogation and direct reflectometric demodulation system. The FBGs had a grating length of $1 \mathrm{~mm}$ (much shorter than the $\mathrm{AE}$ wavelength) according to the condition of $\lambda_{s} / L>>1$ and had central wavelengths of $1553.260 \mathrm{~nm}$ and $1553.250 \mathrm{~nm}$, respectively. Both FBG 1 and FBG 2 were fabricated under the same condition, and their reflection ratios were above $80 \%$. And they were glued to the surface of the plate. As shown in Fig. 1, the output light power from the circulator was transformed by the photodiode into the electrical power which was processed and recorded by the computer. In order to meet the requirement of the interrogation system, the wavelength of the tunable fiber laser was set at $1553.160 \mathrm{~nm}$, which was the left mid-reflection wavelength of the FBGs to achieve the ideal match demodulation. And the output power was chosen as $9 \mathrm{~mW}$. 


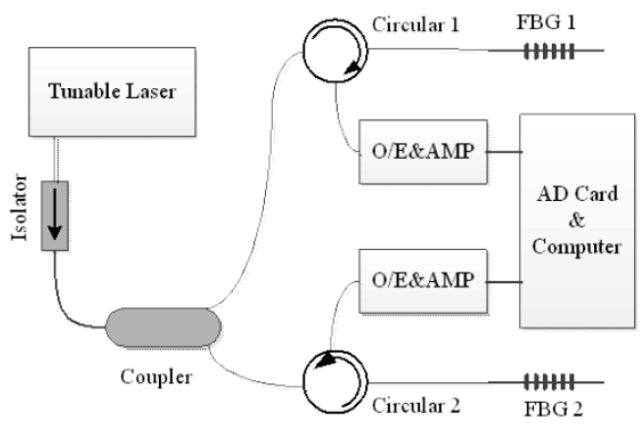

Fig. 1 Experimental setup for the FBG-based AE location sensing system.

Lead break signals were used as simulated $\mathrm{AE}$ signals. The typical waveform of $30^{\circ}$ lead break signal on a marble slab with dimensions $600 \mathrm{~mm} \times$ $400 \mathrm{~mm} \times 5 \mathrm{~mm}$ is shown in Fig. 2(a). The signal recorded was $400 \mathrm{~mm}$ away from the FBG sensor. To determine the arrival time of the simulated $\mathrm{AE}$ signal at a single frequency, we used $100 \mathrm{kHz}$ in the WT analysis. This frequency was chosen because the spectral analysis as shown in Fig. 2(b) showed that this was one of the typical frequencies presented in the captured waveforms. From Fig.2(c), we can see that the WT analysis of the AE signal contains multiple peaks. The first peak represented the arrival time of the $\mathrm{S}_{0}$ mode wave, and the second represented the arrival time of the $A_{0}$ mode wave. Because the $A_{0}$ mode wave propagated much slower than the $\mathrm{S}_{0}$ mode wave and the $\mathrm{A}_{0}$ mode wave had much larger magnitude than the $S_{0}$ mode wave, these two peaks were clearly distinguishable and separated in time. The following peaks indicated the stationary vibration of the plate or the reflective pulses of the signal.

In the $\mathrm{AE}$ location performance, it is important that we use the WT coefficient of the identical mode for the accurate source location. There may be cases where it is difficult to distinguish the two modes in WT data of the AE signal when the AE source is too close to the sensor thus the two modes are not that separated. So an efficient way has to be sought to identify the arrival time of the same mode of an $\mathrm{AE}$ signal. Here, we set a threshold, which was $80 \%$ of the maximum magnitude of each event. Hence, the first peak after the threshold crossing indicates the arrival time of the $\mathrm{A}_{0}$ mode wave traveling with the $\mathrm{A}_{0}$ mode wave group velocity in the plate. In this way, after measuring the group velocity of the $A_{0}$ mode wave at $100 \mathrm{kHz}$, we can locate the $\mathrm{AE}$ source through the WT and threshold analysis.
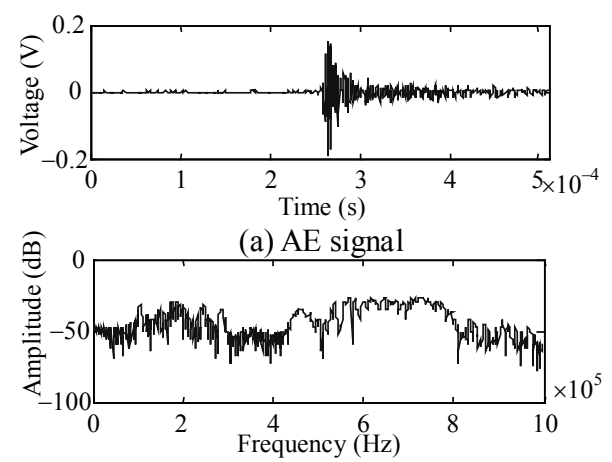

(b) Fourier transform

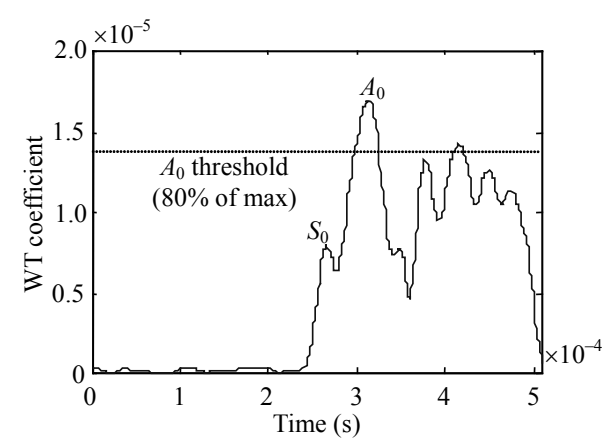

(c) Gabor WT coefficient at 100

Fig. 2 Lead break signal $400 \mathrm{~mm}$ away from the sensor.

100 pencil lead break signals were performed on the marble slab to testify the location method of the WT and threshold analysis. Setting the $y$-direction coordinate of the FBG sensors as zero, the coordinates of FBG 1 and FBG 2 were $(0,0)$ and $(600,0)$, separately. And lead breaks were conducted at $(230,0)$. In the experiments, AE signals were first sensed by the FBG sensors, then transformed into electrical signals and recorded to the computer. Finally, they were analyzed using the WT wavelet and threshold analysis to identify the exact position of the AE source. The result showed that 86 of the 100 events $(86 \%)$ had errors less than $10 \mathrm{~mm}$. In the other 14 events, 9 of them had errors less than $20 \mathrm{~mm}$, and 5 of them had errors less than $30 \mathrm{~mm}$. 


\section{Conclusions}

A novel ultra-short FBG sensor used to detect $\mathrm{AE}$ signals was developed, and the interrogation scheme utilizing the tunable narrow laser was verified. In addition, an improved $\mathrm{AE}$ location method based on the Gabor wavelet transform and threshold analysis was deduced and verified in $\mathrm{AE}$ linear location experiments using the ultra-short FBG sensors. With the new AE location method, we can avoid distinguishing the two modes contained in the AE signal and achieve the arrival time difference of different sensors simply and correctly. Since the $\mathrm{AE}$ detection and $\mathrm{AE}$ source location have the extensive application foreground, the future research will regard the theoretical analysis of the FBG to $\mathrm{AE}$ wave and the improved $\mathrm{AE}$ location algorithm. Although experiments were done on a marble slab, we expect it to be extended to the problems with all thin-plate structures.

\section{Acknowledgement}

The authors gratefully acknowledge the financial support for this work from the Natural Science Foundation of China (Grant No. 61074163) and the Natural Science Foundation of Shandong Province, China (Grant No. ZR2011FQ025).

Open Access This article is distributed under the terms of the Creative Commons Attribution License which permits any use, distribution, and reproduction in any medium, provided the original author(s) and source are credited.

\section{References}

[1] A. Othonos, "Fiber Bragg gratings," Review of Scientific Instruments, 1997, 68(12): 4309-4341.

[2] G. F. Fernando, D. J. Webb, and P. Ferdinand, "Optical-fiber sensors," MRS Bulletin, 2002, 27(05): 359-364.

[3] A. Minardo, A. Cusano, R. Bernini, L. Zeni, and M. Giordano, "Response of fiber Bragg gratings to longitudinal ultrasonic waves," Ultrasonics, Ferroelectrics and Frequency Control, IEEE Transactions on, 2005, 52(2): 304-312.

[4] J. R. Lee and H. Tsuda, "Sensor application of fibre ultrasonic waveguide," Measurement Science and Technology, 2006, 17(4): 645-652.

[5] J. R. Lee and H. Tsuda, "Investigation of a fibre wave piezoelectric transducer," Measurement Science and Technology, 2006,17(9): 2414-2420.

[6] J. R. Lee, S. S. Lee, and D. J. Yoon, "Simultaneous multipoint acoustic emission sensing using fibre acoustic wave grating sensors with identical spectrum," Journal of Optics A: Pure and Applied Optics, 2008, 10(8): 085307.

[7] O. Y. Kwon and Y. C. Joo, "Source location in plates by using wavelet transform of AE signals," Journal of Acoustic Emission, 1998, 16(1-4): S212-S221.

[8] H. Jeong and Y. S. Jang, "Fracture source location in thin plates using the wavelet transform of dispersive waves," Ultrasonics, Ferroelectrics and Frequency Control, IEEE Transactions on, 2000, 47(3): 612-619.

[9] B. Culshaw, G. Thursby, D. Betz, and B. Sorazu,, "The detection of ultrasound using fiber-optic sensors," Sensors Journal, IEEE, 2008, 8(7): 1360-1367.

[10] C. Zhou and Y. Zhang, "Fatigue crack localization with near-field acoustic emission signals," in Proc. SPIE International Society for Optics and Photonics, vol. 8692, pp. 86920L-1-86920L-9, 2013. 